\title{
Subdirectly irreducible distributive nearlattices
}

\author{
Radomir Halǎ̌
}




\title{
SUBDIRECTLY IRREDUCIBLE DISTRIBUTIVE NEARLATTICES
}

\author{
RADOMIR HALAŠ
}

[Received: October 10, 2005]

\begin{abstract}
A nearlattice means a join-semilattice having the property that every principal filter (or a section) is a lattice with respect to a semilattice order. The aim of the paper is to characterize in a simple way subdirectly irreducible distributive nearlattices.
\end{abstract}

Mathematics Subject Classification: 06B05, 06B25, 06A12

Keywords: nearlattice, semilattice, distributivity, subdirect irreducibility

\section{INTRODUCTION}

Algebraic structures being join-semilattices with respect to a naturally induced order relation appear very frequently in algebraic logic. For example, implication algebras, introduced by Abbott in the 1960s, describe algebraic properties of the logical connective implication in a classical propositional logic. Implication algebras have a very nice structure: with respect to the induced order, they are join-semilattices whose principal filters are Boolean algebras.

For various logics of quantum mechanics the corresponding algebraic structures have a semilattice structure with principal filters being special lattices, see, e. g., [1$5,7]$.

This fact motivated I. Chajda and M. Kolařík to describe all $\vee$-semilattices where every principal filter is a lattice not satisfying any additional condition. More precisely, they studied the following structures:

Definition 1. A semilattice $\delta=(S, \vee)$, where for each $a \in S$ the principal filter $[a)=\{x \in S \mid a \leq x\}$ is a lattice with respect to the induced order $\leq$ of $\delta$, is called a nearlattice.

It has been shown [6] that nearlattices can be considered as algebras with one ternary operation. Moreover, nearlattices considered as algebras of type (3) form an equational class: indeed, if $x, y, z \in S$ for a nearlattice $\mathcal{S}$, the element $(x \vee z) \wedge(y \vee z)$ is correctly defined since both $x \vee z, y \vee z \in[z)$ and [z) are lattices. The following proposition has been obtained in [6]: 
Proposition 1 ([6]). Let $\delta=(S, \vee)$ be a nearlattice. Define a ternary operation $m(x, y, z)=(x \vee z) \wedge(y \vee z)$ on $S$. Then $m(x, y, z)$ is an everywhere defined operation and the following identities are satisfied:

(P1) $m(x, y, x)=x$,

(P2) $m(x, x, y)=m(y, y, x)$,

(P3) $m(m(x, x, y), m(x, x, y), z)=m(x, x, m(y, y, z))$,

(P4) $m(x, y, p)=m(y, x, p)$,

(P5) $m(m(x, y, p), z, p)=m(x, m(y, z, p), p)$,

(P6) $m(x, m(y, y, x), p)=m(x, x, p)$,

(P7) $m(m(x, x, p), m(x, x, p), m(y, x, p))=m(x, x, p)$.

Conversely, let $(S, m)$ be an algebra of type (3) satisfying the identities (P1)-(P7). If we define $x \vee y=m(x, x, y)$, then $(S, \vee)$ is a join-semilattice and for each $p \in S$, $([p), \leq)$ is a lattice, where for $x, y \in[p)$ their infimum is $x \wedge y=m(x, y, p)$. Hence $(S, \vee)$ is a nearlattice.

Thus nearlattices similarly to lattices have two faces and we shall alternate in our investigations between them depending on which one is more convenient.

Proposition 2 ([6]). The variety of nearlattices is congruence distributive.

The following notions of distributivity for nearlattices have been introduced in [6]:

Definition 2. Let $\delta=(S, m)$ be an algebra of type (3). We call 8 distributive if it satisfies the identity

(D1) $m(x, m(y, y, z), p)=m(m(x, y, p), m(x, y, p), m(x, z, p))$.

If $(S, m)$ satisfies the identity

(D2) $m(x, x, m(y, z, p))=m(m(x, x, y), m(x, x, z), p)$,

then it is called dually distributive.

It is expected that both notions are in case of nearlattices related. Indeed, one can prove the following statement:

Proposition 3 ([6]). Let $\delta=(S, m)$ be an algebra of type (3) satisfying (P1)(P7). Then the following conditions are equivalent:

(1) 8 is distributive,

(2) 8 is dually distributive,

(3) in the associated semilattice, every principal filter is a distributive lattice.

In spite of the previous description of distributivity for nearlattices, we are able to prove by using very simple arguments that there is only one subdirectly irreducible nearlattice of the variety $\mathfrak{D} N$ of distributive nearlattices, namely, the two element lattice. 


\section{SUBDIRECTLY IRREDUCIBLE DISTRIBUTIVE NEARLATTICES}

Definition 3. A subset $\varnothing \neq I \subseteq S$ of a nearlattice $\delta=(S, \vee)$ is called an ideal if

(I1) $m(x, x, y)=x \vee y \in I$ for all $x, y \in I$,

(I2) $m(x, y, p) \in I$ for all $x, p \in I$ and $y \in S$.

Note that $I$ is an ideal of $\delta$ iff $I$ is closed under suprema and $I$ is a downset of $(S, \leq)$.

Secondly, by a filter of $\delta=(S, \vee)$ is meant a subset $\varnothing \neq F \subseteq S$ closed under imfima which is an upset of $(S, \leq)$.

An ideal $I$ of $(S, \vee)$ is called prime if for all $x, y \in S$, the existence of $x \wedge y$ and $x \wedge y \in I$ yield $x \in I$ or $y \in I$.

Let us mention that the primality of $I$ is equivalent to the following condition:

(*) $\forall x, y, p \in S:(p \in I$ and $(x \vee p) \wedge(y \vee p) \in I) \Rightarrow x \vee p \in I$ or $y \vee p \in I$.

Indeed, if $I$ is prime, then evidently $(*)$ holds. Conversely, assume that $(*)$ holds and let $x \wedge y \in I$ exists. Then putting $p:=x \wedge y$ in $(*)$, we get $x=x \vee p, y=y \vee p$ and $(x \vee p) \wedge(y \vee p)=x \wedge y \in I$. But $(*)$ yields $x=x \vee p \in I$ or $y=y \vee p \in I$ and $I$ is prime.

Clearly, for each $a \in S,(a]=\{x \in S \mid x \leq a\}$ is an ideal of $8=(S, \vee)$ as well as $[a)=\{x \in S \mid a \leq x\}$ is a filter of $\delta=(S, \vee)$. The set $\operatorname{Id}(\delta)$ of all ideals of $\delta$ forms with respect to $\subseteq$ a complete lattice and for each $I \in \operatorname{Id}(\&)$ and $a \in S$, the join $I \vee(a])$ in $\operatorname{Id}(\&)$ is

$$
I \vee(a]=\{z \in S \mid z \leq i \vee a \text { for some } i \in I\} .
$$

The following statement can be considered as a prime ideal theorem for nearlattices:

Theorem 1. Let 8 be a distributive nearlattice, $I$ be an ideal of 8 and $D$ be a filter of 8 . If $I \cap D=\varnothing$, then there is a prime ideal $P \supseteq I$ with $P \cap D=\varnothing$.

PROOF. It is evident that the uniton of a chain of ideals of $\delta$ containing $I$ and having an empty intersection with $D$ is again an ideal of this kind. Hence, applying Zorn's lemma, there are maximal ideals $P \supseteq I$ with $P \cap D=\varnothing$.

Let us prove that $P$ is prime. If not, by $(*)$ there exist $x, y, p \in S$ such that $x \vee p \notin P, y \vee p \notin P$ and $(x \vee p) \wedge(y \vee p) \in P$ for some $p \in P$.

Further, due the to maximality of $P$,

$$
(P \vee(x \vee p]) \cap D \neq \varnothing, \quad(P \vee(y \vee p]) \cap D \neq \varnothing .
$$

Hence there are $a, b \in D$ such that $a \leq r \vee x \vee p$ and $b \leq r \vee y \vee p$ for some $r, s \in P$.

Since $D$ is a filter of $\delta$ and $a, b \in D$, also $r \vee x \vee p \in D$ and $s \vee y \vee p \in D$. Moreover, $r \vee x \vee p, s \vee y \vee p \in[p)$, thus also $(r \vee x \vee p) \wedge(s \vee y \vee p) \in D$. This 
gives also

$$
\alpha=((r \vee p) \vee(x \vee p)) \wedge((s \vee p) \vee(y \vee p)) \in D .
$$

Since all the elements mentioned are above $p$, in view of the distributivity of $[p]$ we derive

$\alpha=((r \vee p) \wedge(s \vee p)) \vee((r \vee p) \wedge(y \vee p)) \vee((x \vee p) \wedge(s \vee p)) \vee((x \vee p) \wedge(y \vee p)) \in D$.

Further, $r, s, p \in P$ yield $r \vee p, s \vee p \in P$ and all the elements in brackets of $\alpha$ belong to $P$. This gives $\alpha \in P \cap D$, which is a contradiction proving that $P$ is prime.

Corollary 1. Let $\delta=(S, \vee)$ be a distributive nearlattice, $a, b \in S, a \neq b$. Then there is a prime ideal $P$ of $\&$ with $a \in P$ and $b \notin P$.

Proof. For $a \neq b$, we have either $(a] \cap[b)=\varnothing$ or $(b] \cap[a)=\varnothing$. The rest is a corollary of Theorem 1 .

Similarly as in distributive lattices, a typical example of a distributive nearlattice is a set-nearlattice:

Definition 4. For a set $S$, define on $\operatorname{Exp}(S)$ a binary operation by

$$
m(X, Y, Z):=(X \cup Z) \cap(Y \cup Z) .
$$

Then $(\operatorname{Exp}(S), m)$ is a distributive nearlattice, called a set-nearlattice on $S$.

Let us show that any distributive nearlattice can be embedded into a set-nearlattice.

Theorem 2. Let $\delta=(S, \vee)$ be a distributive nearlattice, let $\mathcal{P}(S)$ be the set of all prime ideals of 8 . Then the mapping $r: S \rightarrow \operatorname{Exp}(\mathcal{P}(S))$ defined by the formula

$$
r(a):=\{P \in \mathcal{P}(S) \mid a \notin P\}
$$

is an embedding of 8 into $(\operatorname{Exp}(\mathcal{P}(S)), m)$.

PROOF. Due to the previous corollary, $r$ is an injection. So we have to prove that for all $a, b, c \in S$, the relation $r(m(a, b, c))=m(r(a), r(b), r(c))$ is true, or

$$
r((a \vee c) \wedge(b \vee c))=(r(a) \cup r(c)) \cap(r(b) \cup r(c)) .
$$

Let us first show that for all $x, y \in S$,

$$
r(x \wedge y)=r(x) \cap r(y) .
$$

Indeed, (2.2) is equivalent to the statement that for each $P \in \mathcal{P}(S), x \wedge y \notin P$ if and only if $x \notin P$ and $y \notin P$. Then (2.1) can be rewritten into

$$
r(a \vee c) \cap r(b \vee c)=(r(a) \cup r(c)) \cap(r(b) \cup r(c)) .
$$

Evidently, the inclusions $r(a \vee c) \cap r(b \vee c) \supseteq r(c)$ and $r(a \vee c) \cap r(b \vee c) \supseteq$ $r(a) \cap r(b)$ are valid. Thus,

$$
r(a \vee c) \cap r(b \vee c) \supseteq r(c) \cup(r(a) \cap r(b))=(r(a) \cup r(c)) \cap(r(b) \cup r(c)) .
$$


Let us show the converse inclusion. For this, assume $P \in r(a \vee c) \cap r(b \vee c)$, i. e., $a \vee c \notin P$ and $b \vee c \notin P$. Then $P \notin r(a) \cup r(c)$ would lead to $a, c \in P$ and $a \vee c \in P$, which is a contradiction. Thus $P \in r(a) \cup r(c)$ and, analogously, $P \in r(b) \cup r(c)$, which altogether gives

$$
r(a \vee c) \cap r(b \vee c) \subseteq(r(a) \cup r(c)) \cap(r(b) \cup r(c))
$$

and completes the proof.

Corollary 2. Let $\delta=(S, m)$ be a distributive nearlattice, $|S| \geq 2$. Then the identity $p \approx q$ holds in 8 iff $p \approx q$ holds in a 2-element nearlattice $\mathbf{C}_{2}$.

Proof. If $|S| \geq 2$, then $(S, \leq)$ contains a 2-element chain $M$. Moreover, $\mathcal{M}=$ $(M, m)$ is a subalgebra of $\delta$, hence if $p \approx q$ holds in $\delta$, it holds also in $\mathcal{M}$.

Conversely, every set-nearlattice $(\operatorname{Exp}(B), m)$ is isomorphic to a direct product $\mathcal{M}^{|B|}$. Hence if $p \approx q$ holds in $\mathbf{C}_{2}=\mathcal{M}$, it holds also in $(\operatorname{Exp}(B), m)$ and since 8 can be embedded into a set-nearlattice, $p \approx q$ holds in $\delta$.

It is clear that given a nearlattice $\delta=(S, m)$ and $c \in S,[c)$ is a subalgebra of $\delta$. Let us show that for an equivalence $\theta$ on $[c), \theta \in \operatorname{Con}([c), m)$ iff $\theta \in \operatorname{Con}([c), \wedge, \vee)$.

Since $\wedge$ and $\vee$ are both term operations of $\delta$, the implication $(\Rightarrow)$ is immediate. Conversely, if $\theta \in \operatorname{Con}([c), \wedge, \vee)$ and $(a, b) \in \theta,(x, y) \in \theta$ and $(u, v) \in \theta$ for some $a, b, x, y, u, v \geq c$, then

$$
m(a, x, u)=(a \vee u) \wedge(x \vee u) \equiv_{\theta}(b \vee v) \wedge(y \vee v)=m(b, y, v),
$$

verifying $\theta \in \operatorname{Con}([c), m)$.

Now we are ready to describe subdirectly irreducible members of the variety $\mathscr{D} N$ of all distributive nearlattices. Denote by $\theta(a, b)$ the principal congruence generated by $(a, b)$.

Theorem 3. The variety $\mathfrak{D} N$ has only one subdirectly irreducible member, namely $\mathrm{C}_{2}$.

Proof. Let $\delta=(S, m)$ be a subdirectly irreducible distributive nearlattice, and assume that $\mu=\theta(a, b)$ is its monolith. We may assume $a \neq b$. Then $(a, b) \in \mu$ yields $(a, a \vee b) \in \mu, a \neq a \vee b$. Thus $\theta(a, a \vee b) \neq \omega$ and $\theta(a, b) \subseteq \theta(a, a \vee b)$.

Conversely, $(a, a \vee b) \in \mu$ gives $\theta(a, a \vee b) \subseteq \mu$ and altogether $\theta(a, a \vee b)=$ $\theta(a, b)=\mu$.

Let now $c \in S$ be given, and denote $\mu_{c}=y \cap[c)^{2}$ the congruence induced on a subalgebra $[c)$. If $y_{c} \neq \omega$, then $y_{c}$ is the least nonzero congruence on the distributive lattice $([c), \wedge, \vee)$. Indeed, if $\theta \in \operatorname{Con}([c), \wedge, \vee)$ and $\theta \neq \omega$, then $\theta \in \operatorname{Con}([c), m)$. Since the variety $\mathscr{D} N$ is congruence distributive, $\mathscr{D} N$ has the congruence extension property. Thus there is $\psi \in \operatorname{Con} \delta$ with $\theta=\psi \cap[c)^{2}$. Moreover, $\theta \neq \omega$ yields $\psi \neq \omega$, hence $\mu \subseteq \psi$ and $\mu_{c}=\mu \cap[c)^{2} \subseteq \psi \cap[c)^{2}=\theta$, proving that $\mu_{c}$ is the least nonzero congruence on $([c), m)$, and hence the monolith on $([c), \wedge, \vee)$. Now, since $\mu_{a} \neq \omega, \mu_{a}$ is the monolith on the distributive lattice $([a), \wedge, \vee)$, and hence 
$|[a)|=2$, i.e. $[a)=\{a, a \vee b\}$. This yields $a \vee b=1$, the greatest element of $(S, \leq)$ (otherwise $[a) \neq\{a, a \vee b\}$ ).

Analogously, for every $c \leq a, \mu_{c} \neq \omega$ is the monolith on $[c)$, hence for $|[c)|=2$ we obtain $c=a$. Then the nearlattice $\delta$ is of the form shown on Figure 1 .

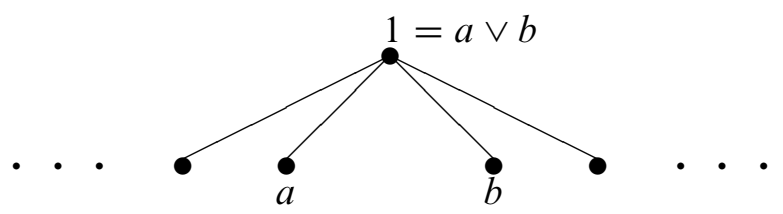

FIGURE 1.

It is easily seen that $\theta(1, a)=\{(1, a),(a, 1)\} \cup \omega$, so $\mu=\theta(1, a)$ forces $S=$ $\{1, a\}$, which completes the proof.

\section{ACKNOWLEDGEMENT}

The financial support by Grant MSM 6198959214 of the Czech Government is gratefully acknowledged.

\section{REFERENCES}

[1] Аввотт, J. C.: Semi-boolean algebra, Mat. Vestnik, 4 (1967), 177-198.

[2] Аввотт, J. C.: Orthoimplication algebras, Studia Logica, 35 (1976), 173-177.

[3] CHAJdA, I.: An extension of relative pseudocomplementation to non-distributive lattices, Acta Sci. Math. (Szeged), 69 (2003), 401-406.

[4] Chajda, I., Halaš, R., AND LÄNGer, H.: Orthomodular implication algebras, Internat. J. Theoret. Phys., 40 (2001), 1875-1884.

[5] Chajda, I., Halaš, R., AND LÄNGER, H.: Simple axioms for orthomodular implication algebras, Internat. J. Theoret. Phys., 43 (2004), 911-914.

[6] Chajda, I. And KolaŘík, M.: Nearlattices, Discrete Math., submitted.

[7] Chajda, I. ANd RAdeleczKi, S.: On varieties defined by pseudocomplemented nondistributive lattices, Publ. Math. Debrecen, 63(4) (2003), 737-750.

[8] GRÄTZER, G.: Lattice Theory, Birkhäuser Verlag, Basel, 1978.

\section{Author's address}

\section{Radomir Halaš:}

Palacký University Olomouc, Tomkova 40, 77900 Olomouc, Czech Republic

E-mail address: halas@inf.upol.cz 